FORUM

\title{
El rol de Turdus falcklandii (Aves: Passeriforme) como dispersor de plantas invasoras en el archipiélago de Juan Fernández
}

\author{
The role of Turdus falcklandii (Aves: Passeriforme) as disperser of invasive plants in the \\ Juan Fernández Archipelago
}

\section{CECILIA SMITH-RAMÍREZ1,2, GISELLA ARELLANO ${ }^{1}$, ERIN HAGEN ${ }^{3}$, RODRIGO VARGAS ${ }^{4}$, JESSICA CASTILLO ${ }^{1,5}$ \& ALEJANDRO MIRANDA 6}

\author{
${ }^{1}$ Instituto de Ecología y Biodiversidad (IEB), Las Palmeras 3425, Ñuñoa, Santiago \\ 2Instituto de Manejo Forestal, Universidad Austral de Chile, isla Teja s/n, Valdivia \\ ${ }^{3}$ Island Conservation, Las Urbinas 53, of. 42, Santiago, Chile \\ ${ }^{4}$ Walbau-Institut, Universitat Freiburg, Tennenbacher Straße 479106 Freiburg Alemania \\ ${ }^{5}$ Facultad de Ciencias Forestales y Recursos Naturales, Universidad Austral de Chile, isla Teja s/n, Valdivia \\ ${ }^{6}$ Universidad de La Frontera, Departamento de Ciencias Forestales, Montevideo s/n, Temuco, Chile
}

*Autor correspondiente: csmith@willnet.cl

\begin{abstract}
RESUMEN
La Reserva Mundial de la Biósfera, Archipiélago de Juan Fernández ( $\left.33^{\circ} \mathrm{S}\right)$ presenta la flora insular con mayor endemismo $\mathrm{m}^{-2}$ en el mundo. Tres especies de arbustos introducidos invaden y desplazan la vegetación nativa de Juan Fernández, especialmente en la isla Robinson Crusoe. Estas especies, Aristotelia chilensis (maqui, introducido en 1894), Rubus ulmifolius (mora, introducido en 1927) y Ugni molinae (murta, introducido a principios del siglo $\mathrm{XX}$ ), presentan frutos carnosos que son dispersados principalmente por Turdus falcklandii (zorzal). En este trabajo se plantean y responden cinco preguntas clave, sobre: la capacidad actual de expansión de las plantas invasoras; el efecto del zorzal sobre la dispersión y germinación de estas plantas; y el posible efecto de controlar zorzal tanto sobre la expansión de las plantas invasoras como las nativas. Se concluye que la interacción entre el zorzal y los arbustos invasores ha sido clave para explicar su progresiva expansión, además de los rasgos de historia de vida de las plantas invasoras. Se plantea que el control o erradicación de esta ave podría ser una herramienta adecuada para desacelerar el avance de las plantas invasoras. Se recomienda hacer estudios detallados que permitan tomar decisiones sobre la efectividad de controlar en determinada cantidad y tiempo, o erradicar la población de zorzal de la isla Robinson Crusoe como medida de manejo. Además, se recomienda realizar estudios que permitan planificar el control de las fitoplagas en diferentes etapas del ciclo de vida de estas. De no tomarse prontas y efectivas medidas de control de la expansión de maqui, mora y murta se prevé una pérdida considerable de la biodiversidad terrestre de Robinson Crusoe en un mediano plazo.
\end{abstract}

Palabras clave: Aristotelia chilensis, interacción biológica, plantas invasoras, Rubus ulmifolius, Ugni molinae.

\begin{abstract}
The Juan Fernandez Archipelago World Biosphere Reserve ( $\left.33^{\circ} \mathrm{S}\right)$ contains the highest density of insular floral endemism in the world. Currently three invasive woody plant species threaten the conservation of the vegetation of Juan Fernández, particularly on Robinson Crusoe Island. The introduced plant species, Aristotelia chilensis (maqui), Rubus ulmifolius (mora) and Ugni molinae (murta), have fleshy fruits that are dispersed primarily by a native bird, the Austral Thrush (Turdus falcklandii). We explore five key scientific questions to guide management decisions, regarding the current expansion capacity of invasive plants, the influence of the Austral Thrush on dispersal and germination of invasive plants in forest gaps, and the possible effects of Austral Thrush control on the expansion of native and invasive plants. We conclude that the interaction between the Austral Thrush and invasive weeds, in addition to the life history characteristics of the invasive species, has been a defining characteristic of the success and speed of the plant invasion, and therefore control or eradication of the Austral Thrush could be a useful tool to slow invasive plant advances. However, we recommend additional detailed studies that would guide decision-making regarding Austral Thrush control and/or eradication effectiveness on Robinson Crusoe Island, in addition to studies of invasive plant control in various life history stages. If immediate and effective control measures are not taken to prevent the expansion of maqui, mora and murta, we foresee a considerable loss of terrestrial biodiversity from Robinson Crusoe Island in the medium term.
\end{abstract}

Key words: Aristotelia chilensis, biological interaction, invasive plant species, Rubus ulmifolius, Ugni molinae. 


\section{INTRODUCCIÓN}

Las especies invasoras son consideradas una de las principales amenazas para la biodiversidad a nivel mundial (Davis 2003). En la actualidad, este fenómeno es considerado un componente significativo del cambio biótico global (Sala et al. 2000) y uno de los grandes motores de la extinción de especies (Money \& Hobbs 2000). Este fenómeno es de especial importancia en ecosistemas insulares, en donde existe amplia evidencia del progresivo reemplazo que ha sufrido la flora vascular nativa producto de la introducción de especies exóticas (Fritts \& Rodda 1998, Bergstrom \& Chown 1999, Sax et al. 2002, Denslow 2003, Gaston et al. 2003, Castro et al. 2007, Baret et al. 2008, Kueffer et al. 2010), particularmente en islas del Océano Pacífico (Daehler et al. 2004, Castro \& Jaksic 2008).

Se ha establecido que las interacciones mutualistas planta-animal, tales como la dispersión de semillas, pueden jugar un rol importante en los procesos invasivos al permitir que ciertas especies se tornen invasivas (Richardson et al. 2000, Williams 2006, Kueffer et al. 2009). El escenario en que dos especies invasivas se potencian (Simberloff \& von Holle 1999) no es tan complicado, en términos de manejo, como lo es el escenario en que se ve facilitada o promovida la invasión por una o varias especies nativas (Gosper et al. 2005, Buckley et al. 2006). Cuando los tomadores de decisión se enfrentan a este último escenario, las opciones de manejo se complejizan debiendo favorecer la permanencia de la mayoría de las especies nativas, en desmedro de otras, o se llega a una inmovilidad ante la dificultad de tomar decisiones.

Probablemente dos de los casos más graves del deterioro de la biodiversidad mundial producto de una interacción mutualista de un ave nativa con plantas introducidas, ocurren en Chile en el Archipiélago de Juan Fernández (AJF) y en el archipiélago de Galápagos, Ecuador (Atkinson et al. 2010). Además en la isla Réunion, Francia, ha sido reportada la dispersión de varias especies de plantas invasoras, aunque no por aves nativas sino introducidas (Mandon-Dalger 2004).

El AJF se encuentra a $670 \mathrm{~km}$ de Chile continental y está compuesto por tres islas, Robinson Crusoe (RC), Santa Clara y Marinero
Alejandro Selkirk (AS). El AJF posee el mayor grado de endemismo vegetal en Chile (63.4\%, Danton 2004) y el más alto por metro cuadrado en islas a nivel mundial (Stuessy 1992, Arroyo 1999). La mayor parte del AJF se encuentra protegido bajo la categoría de Parque Nacional, el cual fue creado en 1935. En 1977, el Parque Nacional fue nombrado Reserva de la Biosfera. En la actualidad, el archipiélago alberga 503 especies de plantas introducidas $(71 \%$ del total de especies, Danton \& Perrier 2006). No obstante, solo tres taxa leñosos son los que han afectado severamente la persistencia del bosque nativo (Cuevas \& Van Leersum 2001, Greimler et al. 2002, Dirnböck et al. 2003, Cuevas et al. 2004). Estas especies son: Aristotelia chilensis (Molina) Stunz, Elaeocarpaceae (maqui), Rubus ulmifolius Schott, Rosaceae (mora) y Ugni molinae Turcz., Myrtaceae (murtilla o murta). Las tres especies presentan frutos carnosos, cuyas semillas son dispersadas por Turdus falcklandii (King), Turdidae (zorzal). Se ha planteado que esta interacción ha impulsado el éxito de estas malezas, afectando directamente la composición, estructura y regeneración de la vegetación nativa en la isla RC (Vargas et al. 2010).

El zorzal fue registrado por primera vez en el AJF en 1741, por el navegante inglés George Anson (Swenson et al. 1997). Dada la baja probabilidad de que haya sido llevado intencionalmente en barco, creemos que se trata de una especie nativa. Análisis de variabilidad genética basados en ADN mitocondrial realizados a individuos de zorzal de RC, AS y Chile continental, muestran que no hay diferencias genéticas entre estos, descartando la posibilidad de que sea una especie o sub especie endémica del archipiélago (González et al., datos no publicados). Sin embargo, parece haber cierta diferenciación genética del zorzal de AS respecto al zorzal de RC y continente (González et al. datos no publicados).

Por otra parte, el maqui habría arribado a RC en 1864, ya Carl Skottsberg señalaba en 1953 que esta especie formaba un matorral que se internaba en el bosque de tierras bajas limitando su expansión (Dirnböck et al. 2003). La murta fue registrada en 1892 en RC por Friedrich Johow (Dirnböck et al. 2003), mientras que la mora habría sido introducida a esta misma isla en 1927 (Looser 1927). 
En el Plan de Manejo del AJF del año 1976 se considera la mora como especie plaga, estableciendo que de no ejecutarse medidas de control, podría tornarse invasiva. En ese mismo informe, hace 34 años, no se menciona la murta como plaga (CONAF 1976). A su vez, AS cuenta con 128 ha de especies invasoras (CONAF 2009), maqui habría llegado a esta isla hace 25 años y más recientemente mora y murta (Guardaparques AJF, com. pers. 2010).

El objetivo de este trabajo es presentar antecedentes sobre la interacción mutualista entre zorzal y tres arbustos invasores en el AJF y las consecuencias negativas de este mutualismo, que pueden llevar a tomar medidas de control o erradicación del ave. Buckley et al. (2006) plantean que hay seis rasgos de interés, desde un nivel específico a la escala de paisaje, que deben ser estudiados en este tipo de mutualismos para determinar sus efectos negativos o positivos, y que pueden ser útiles en manejo. Estos son: los rasgos de los frutos, los rasgos de los frugívoros, el patrón de establecimiento de la o las plantas invasoras, la estructura del paisaje y la determinación de modelos de expansión de los interactuantes. Debido a que generalmente las interacciones mutualistas son difusas y compuestas por varias especies, resulta compleja la determinación de estos parámetros al momento de tomar decisiones de manejo. Sin embargo, en el AJF la interacción de dispersión de semillas se simplifica, existiendo solo un agente dispersor principal, el zorzal, el cual dispersa un número acotado de especies con frutos carnosos. Por lo cual, dentro de la complejidad de los estudios propuestos por Buckley et al. (2006), el que presentamos resulta ser un ensamble relativamente simple de analizar.

Proponemos en este trabajo cinco preguntas genéricas clave que deben ser enfrentadas para determinar el grado de sinergismo de un ave mutualista con plantas introducidas, de tal forma de determinar la efectividad de controlar o erradicar temporalmente al ave como una medida de control de las plantas invasoras. Las respuestas a estas preguntas incluyen algunos de los rasgos de interés planteados por Buckley et al. (2006), aunque no explícitamente. Por ejemplo, el estudio de los rasgos de los frutos de la planta (ancho del fruto, palatabilidad, preferencia) y del mutualista (ancho de la comisura del pico) están implícitos en la determinación del número de semillas en fecas. Es decir, si se encuentran semillas de la especie de interés en las fecas del ave mutualista, es porque la morfología de esta y el fruto así lo permiten.

Las preguntas que planteamos son: (1) ¿Existe en el tiempo un aumento demográfico de ambos mutualistas o el sistema está estacionario? Los modelos de mutualismo suponen un crecimiento ilimitado de los interactuantes quienes potencian su crecimiento poblacional entre sí, hasta que se alcanza una capacidad de carga (Graves et al. 2006). Si esta capacidad de carga se hubiese alcanzado en la isla RC, la población de las especies interactuantes estaría casi estacionaria. Para contestar esta pregunta se analizaron antecedentes sobre cambios temporales en cobertura de las plantas invasoras y censos demográficos del ave. Si el sistema está estacionario, la urgencia de manejo podría ser baja, a diferencia de si está en expansión. (2) ¿Cuál es la proporción de la lluvia de semillas de las plantas invasoras que es aportada por el o los dispersores en sitios claves de germinación y establecimiento? En general, la dispersión de frutos carnosos por viento es escasa, pero en la isla $\mathrm{RC}$ podría ser alta, dado los fuertes vientos existentes. Solo las semillas de especies invasoras dispersadas en claros de bosque o roqueríos podrán germinar, dado que son sombra intolerantes. La respuesta a esta pregunta permitirá saber si el control o erradicación del ave es una medida eficiente para disminuir la lluvia de semillas en claros. (3) ¿La germinación de las semillas de las plantas invasoras es afectada por su paso a través del tracto digestivo del ave mutualista? La respuesta a esta pregunta permitirá resolver si el ave daña, no afecta o facilita, y en qué medida afecta la germinación de las especies invasoras. (4) ¿Cambiará a nivel comunitario y de paisaje la abundancia y distribución de las especies de plantas invasoras dispersadas por el ave mutualista, si este es controlado o erradicado? (5) $\mathrm{Si}$ el ave es controlado o erradicado ¿producirá cambios negativos en los interactuantes que no sean las plantas introducidas? 
A continuación analizamos los antecedentes existentes en relación a estas preguntas en el sistema constituido por el zorzal y tres especies de plantas invasoras en el AJF. Este análisis nace de la necesidad de organismos del Gobierno de contar con información científica sobre el posible daño que pudiera causar el zorzal a la biodiversidad del AJF. Si bien la información entregada consiste principalmente una revisión, se entregan además, datos en el texto, Tablas y Figuras. Estos datos forman parte de un programa de investigación sobre restauración ecológica en el AJF, muchos de ellos están en proceso de publicación por los autores de este trabajo. Esta información ayudará al lector a entender los planteamientos entregados.

\section{1. ¿EXISTE EN EL TIEMPO UN AUMENTO DEMOGRÁFICO DE AMBOS MUTUALISTAS?}

\section{Antecedentes}

La isla RC, con una superficie de 4.794 ha, presenta en la actualidad 2.309 ha (48 \%) de cobertura de vegetación leñosa (Fig. 1). Mediante la clasificación supervisada de una imagen satelital WorldView-2 (resolución espacial de $2 \mathrm{~m}$ ), obtenida en octubre de 2010, se cuantificó la superficie de las principales coberturas de vegetación. Díaz (2012) determinó que los matorrales invasores de maqui y mora cubren aproximadamente 805.9 ha (Fig. 1) en sectores ubicados en diferentes altitudes, incluyendo el interior de fragmentos de bosque nativo. Las áreas ocupadas casi exclusivamente por murta, cubren aproximadamente 115.9 ha (Fig. 1) en zonas de alta pendiente, usualmente en filos de cerros y laderas (Díaz 2012). El bosque nativo remanente cubre 1014.8 ha (Fig. 1) con dominancia de luma (Nothomyrcia fernandeziana (Hook. et Arn.) Johow), canelo (Drimys confertifolia Phil.), naranjillo (Fagara mayu (Bertero ex Savi) Engler) y helechos nativos (Díaz 2012). El resto de la vegetación corresponde a herbazales que presentan una superficie de 769.5 ha, plantaciones forestales, roca y suelo erosionado.
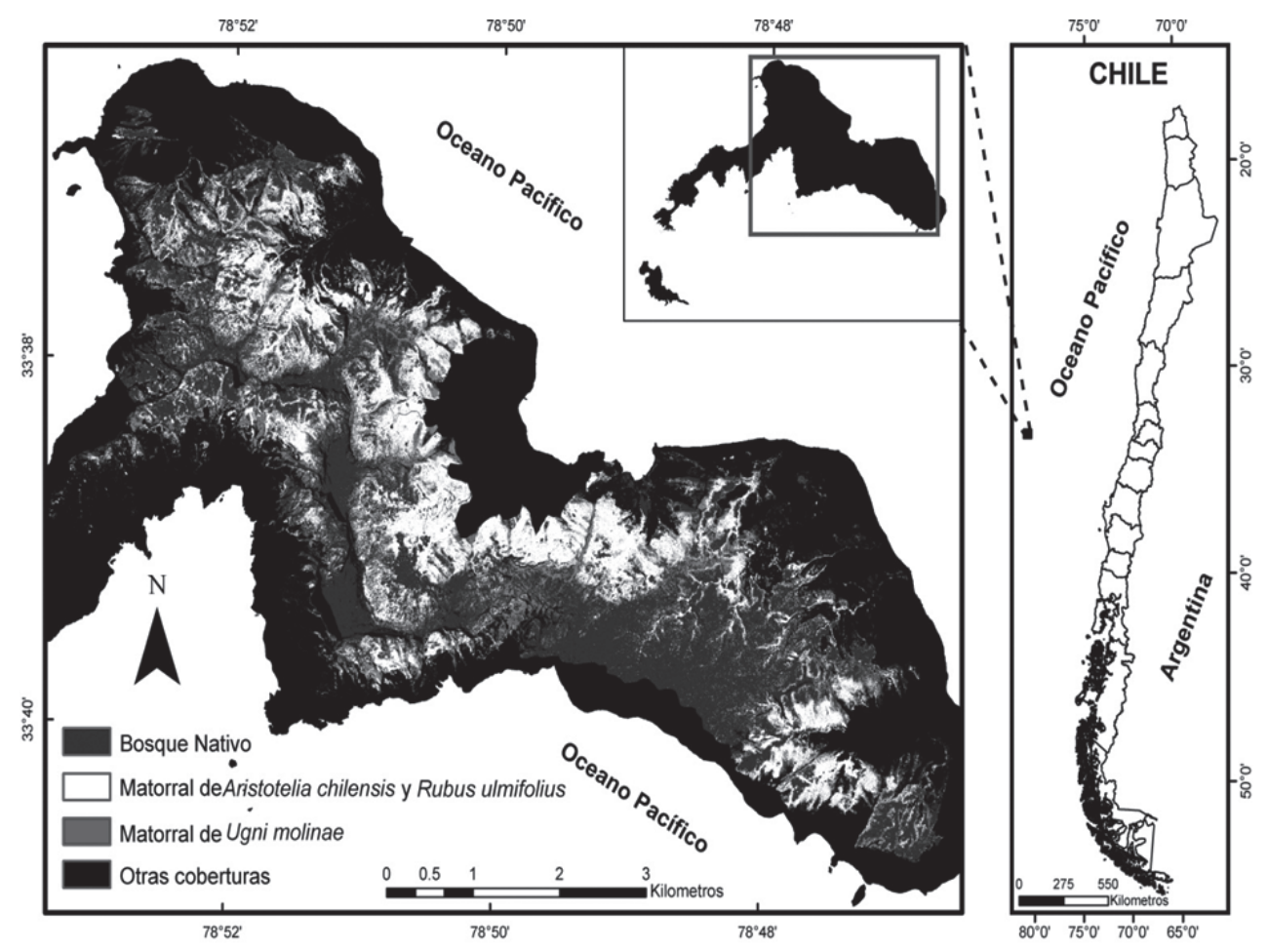

Figura 1: Cobertura de vegetación de especies nativas e introducidas en la isla Robinson Crusoe, Juan Fernández, Chile.

Cover by native and introduced plant species on Robinson Crusoe Island, Juan Fernández, Chile. 


\section{Aumento demográfico de plantas invasoras}

La dinámica de colonización de maqui y mora ocurre a través de dos procesos independientes (Arellano 2012, Díaz 2012): i) la colonización en claros de bosque; y ii) el "retroceso" del borde del bosque original. Los deslizamientos de tierra y la caída natural de los árboles nativos producen claros en el bosque. Los claros de dosel son lugares donde se interrumpe la continuidad del dosel y la luz natural alcanza el sustrato, proceso que resulta fundamental para la regeneración del bosque (Hubbell \& Foster 1986, Yamamoto 1992, Brokaw \& Busing 2000, Dalling \& Hubbell 2002). En el bosque de la isla $\mathrm{RC}$, al igual que en otros bosques, los claros y sus zonas de transición albergan mayor regeneración arbórea y de riqueza de especies vasculares que las zonas bajo dosel (Elias \& Dias 2009, Vargas et al. 2010, Arellano 2012). Los claros detectados en el sector de Plazoleta el Yunque (PEY) de la isla RC (ca. 30 ha de bosque) presentan un rango de tamaño de 13 a $350 \mathrm{~m}^{2}$, con un promedio de $100 \mathrm{~m}^{2}$. Estos claros constituyen entre el 8 al $25 \%$ del paisaje forestal nativo del sector según sea la fase de apertura o cierre en que se encuentren (Vargas et al. 2010, Arellano 2012). Las densidades de plántulas de maqui y mora se asocian positivamente al tamaño de los claros en isla RC (Arellano 2012), no obstante, claros de tamaño menor a $50 \mathrm{~m}^{2}$ presentan una muy baja o nula, regeneración de especies invasoras (Arellano 2012). Ningún estudio de dinámica vegetacional ha sido llevado a cabo en AS.

En el bosque de PEY en RC, en el período de otoño-invierno del 2010 al 2011, se produjeron cinco claros (superficie total 728.8 $\mathrm{m}^{2}$ ) en un sector de 7.5 ha, relativamente plano y expuesto al viento nor-este que rebota desde la meseta del cerro El Yunque. Es decir, 0.0729 ha $(0.97 \%$ de la superficie) entraron a fase de claro en las 7.5 ha de PEY en un año. $\mathrm{Si}$ extrapoláramos este valor de PEY a todo el bosque de RC, donde aún permanecen alrededor de 1.015 ha de bosque nativo (Fig. 1 , Tabla 1), se podría predecir que cerca de 10 ha de bosque son susceptibles de ser invadidas anualmente a través de la dinámica de claros. Por otra parte, Díaz (2012) mediante el análisis de imágenes satelitales encontró que el avance de maqui y mora es de 0.4 ha año-1 en pastizales y bordes de bosque, y el avance de murta sería de 0.5 ha año-1, especialmente en pastizales y roqueríos. Bosques tropicales ubicados entre 0-20 grados de pendiente en la Guayana Francesa y en Panamá, entran anualmente entre 0.64-1.33 \% (Van der Meer \& Bongers 1996) a 1-2 \% en fase claro (Hartshorn 1990), similar a lo encontrado en PEY.

Si bien la apertura de claros no implica necesariamente que sean invadidos, sí existe un alto potencial, dado que casi todos los claros están a distancias menores a $100 \mathrm{~m}$ del matorral invasivo productor de propágulos. La distancia de dispersión de semillas producida por el género Turdus es conocida, no sobrepasando los $100 \mathrm{~m}$ (Debussche et al. 1982, Théry 1989). Turdus merula en España vuela menos que 30 $\mathrm{m}$ antes de percharse y defecar las semillas del Prunus mahaleb L. (cerezo Mahaleb), sin embargo, $4.3 \%$ de su vuelo ocurre más allá de los $30 \mathrm{~m}$ (Jordano \& Schupp 2000). A esta distancia máxima de vuelo casi todos los claros de la isla RC tienen el potencial de ser invadidos, ya que el bosque endémico se encuentra inmerso en una matriz de plantas invasoras. Se estima que seis a siete años después de la llegada de los propágulos de los arbustos invasores a los claros se produce el cierre del dosel por estos y la exclusión de las especies nativas que son sombra intolerantes (Guardaparques AJF, com. pers. 2010).

Se desconoce la dinámica de avance de la invasión mediada por el segundo proceso de colonización, referido al retroceso del bosque que es producido por el "empuje" del matorral adyacente compacto de maqui y mora; no obstante se estima que es un proceso de avance lento (Díaz 2012). En el borde de invasión probablemente existiría competencia por nutrientes y luz, especialmente en el caso de mora que trepa y cubre los árboles nativos (Dirnbock et al. 2003). Esta suma de factores generaría cambios en las condiciones ambientales del suelo debido a un probable aumento del consumo hídrico asociado a una alta competencia intra e inter-específica producida por las invasoras.

Honeyman et al. (2005) mencionan claramente el proceso de reemplazo del bosque endémico de RC por invasoras: "Es factible sentenciar que salvo algunos lugares muy protegidos, los bosques nativos de la isla RC podrían desaparecer cuando todos ellos lleguen a la próxima etapa de desmoronamiento. Esto 
se explica en el siguiente proceso: (i) se forma un claro en el bosque al caer un árbol dominante de gran diámetro de copa. (ii) el área es invadida por plantas plagas impidiendo la regeneración natural. (iii) Esto ocurre en varios sectores, se pierde el continuo del bosque y comienza la fragmentación. Cambia la condición microambiental, las especies incapaces de competir desaparecen y baja la biodiversidad. (iv) Años después comienza el desmoronamiento paulatino de las áreas intermedias de bosque original y los bosques quedan aislados en áreas pequeñas sin dinámica regenerativa. v) Se forma un matorral denso con manchas de bosque nativo y árboles emergentes. vi) Finalmente, dominan las especies invasoras y el bosque nativo se pierde". A su vez, Dirnböck et al. (2003) señalan que la mitad del bosque endémico actual, presenta una probabilidad $>60 \%$ de ser invadido o reemplazado en un plazo de ochenta años. Por otra parte, Smith-Ramírez \& Arellano (2012) muestran una alta fragmentación del bosque fernandeziano, quedando inmerso en una matriz de plantas invasoras leñosas y secundariamente en un matriz de herbáceas y suelo desnudo. La mayor parte de la superficie del bosque endémico se encontraría representado por fragmentos menores a 15 ha (Smith-Ramírez \& Arellano 2012).

\section{Cambios demográficos del ave mutualista}

Estimaciones poblacionales del zorzal en $\mathrm{RC}$ realizadas por Hahn et al. (2006) en el verano del 1994-1995 y del 2001-2002 muestran una población de 6000 y 3000 individuos, respectivamente. A fines de los años $90 \mathrm{y}$ principios del 2000, la Corporación Nacional Forestal (CONAF) con apoyo del Servicio Agrícola Ganadero (SAG) hicieron un concurso de caza para controlar la población del principal dispersor de las especies de arbustos invasores, premiando a quienes cazaran la mayor cantidad de zorzales. El número de zorzales muertos fue cuantificado por pares de patas, entregados a un funcionario del SAG. Así, 1404 individuos (23.4 $\%$ de la población total) de zorzal fueron cazados en un período de tres años. Probablemente la caza fue mayor a este número, no pudiendo muchas veces encontrarse la evidencia de aquellos que caían en el matorral de invasoras, en el bosque, mar o sitios inaccesibles. Este control puede explicar la disminución poblacional (en un $50 \%$ ) en el verano 2001-2002 encontrada por Hahn et al. (2006) respecto al año 1994-1995. A modo de comparación, en la isla AS, donde no hubo caza, Hahn et al. (2006) encontraron una población estable de 1000 ejemplares en cada uno de los mismos períodos de muestreo realizados en RC. Estimaciones poblacionales más recientes en RC (LópezCallejas \& Estades com. pers. 2011), realizadas con un método de análisis de los datos distinto al de Hahn et al. (2006), determinaron que el año 2005 había 3381 individuos y el año 2006 la población habría aumentado a 4832 individuos (Tomasevic et al. 2006).

En conclusión, dados los antecedentes planteados la población de los mutualistas interactuantes no permanece estacionaria, sino que continúa en aumento sostenido.

\section{2. ¿CUÁL ES LA PROPORCIÓN DE LA LLUVIA DE}

SEMILLAS DE LAS PLANTAS INVASORAS QUE ES APORTADA POR EL O LOS DISPERSORES EN SITIOS CLAVE DE GERMINACIÓN Y ESTABLECIMIENTO?

El viento es probablemente el principal agente dispersor de semillas en el AJF. El $84.5 \%$ de las especies nativas de RC son anemófilas (Bernardello et al. 2006). El zorzal es el principal dispersor animal en el AJF, tanto de especies introducidas como nativas. Esta especie tiene una participación reconocida en la dispersión de semillas en el continente, siendo un taxón clave en la reproducción del bosque templado (Armesto et al. 1996). En total, 25 especies nativas de RC y AS (18 y 7 especies, respectivamente) presentan síndrome de endozoocoría. De las 18 especies de $\mathrm{RC}$ con este síndrome, probablemente solo 11 serían dispersadas por zorzal en $\mathrm{RC}$ y solo cinco en AS (Tabla 2). Siete de estas 25 especies con frutos endozoócoros tienen frutos menores a $1.5 \mathrm{~mm}$ de diámetro (por ejemplo, los frutos de Gunnera: Gunneraceae), los cuales tienen baja probabilidad de ser dispersados por aves. A pesar de que proporcionalmente la flora que dispersa el zorzal es muy reducida en relación a la flora nativa total del archipiélago (213 especies nativas), se ha registrado que dispersa dos de las tres especies arbóreas nativas más abundantes de RC, estas son, luma y canelo (Skottsberg 1928, Cuevas \& Vargas 2006; Tabla 2).

Cachudito o cachitoro (Anairetes fernandenzianus, Philippi), un ave endémica 
TABLA 1

Claros generados en el bosque de Plazoleta El Yunque, isla Robinson Crusoe, en el periodo 30 de julio del 2010 a 1 de julio del 2011. *: Expansión de claro.

Forest gaps of Plazoleta el Yunque, Robinson Crusoe Island, formed between July 30, 2001 and July 1, 2011. *: Gap expansion.

\begin{tabular}{|c|c|c|c|c|c|}
\hline $\begin{array}{l}\text { Año de } \\
\text { generación }\end{array}$ & $\begin{array}{l}\text { Mes de creación de } \\
\text { claro }\end{array}$ & $\begin{array}{l}\text { Árbol creador de } \\
\text { claro }\end{array}$ & Radio 1 (m) & Radio 2 (m) & $\begin{array}{l}\text { Área } \\
\left(\mathrm{m}^{2}\right)\end{array}$ \\
\hline 2010 & Agosto & $\begin{array}{c}\text { Myrceugenia } \\
\text { fernandeziana }\end{array}$ & 5.5 & 12.0 & 207.37 \\
\hline 2011 & Marzo-julio & $\begin{array}{l}\text { Myrceugenia } \\
\text { fernandeziana }\end{array}$ & 6.5 & 6.5 & 132.75 \\
\hline 2011 & Abril-junio & $\begin{array}{l}\text { Myrceugenia } \\
\text { fernandeziana }\end{array}$ & 5.7 & 7.4 & 132.53 \\
\hline 2011 & Marzo-junio & Drimys winterii & 3.5 & 3.2 & 35.19 \\
\hline 2011 & Febrero-marzo & Fagara mayu & 11.0 & 6.2 & 214.28 \\
\hline 2011 & Julio & Two Fagara mayu & $4.99-5.09$ & $0.85-4.24$ & $6.66^{\star}$ \\
\hline
\end{tabular}

de RC, también consume ocasionalmente frutos del árbol endémico Juan Bueno (Rhaphithamnus venustus (Phil.) Rob.) (J. Meza, CONAF com. pers. 2010). Cachitoro es una especie preferentemente insectívora, que tiene poca movilidad y un tamaño reducido, lo cual limitaría la ingestión de la mayoría de los frutos de RC (Hahn 2006). La falta de otras aves dispersoras hace pensar que el zorzal fue una especie clave en mantener la dinámica vegetacional de la isla. Con la llegada de las especies invasoras, su rol mutualista con especies nativas se interrumpió, transformándose desde una interacción inicialmente positiva a una interacción negativa, catalizadora de la dispersión de plantas exóticas invasivas, que excluyen a las especies nativas. Aunque el zorzal continúe actualmente dispersando en otoño las semillas de las especies arbóreas nativas, estas no llegarían a formar individuos adultos, producto de que: (1) el lugar donde son dispersadas es un continuo de maqui, mora y/o murta o (2) el rápido crecimiento de las invasoras las suprimirá. Este proceso ocurrirá con la excepción de los pocos lugares donde aún queda bosque nativo sin mayor presencia de malezas, o donde haya claros pequeños, $\leq 50 \mathrm{~m}^{2}$ en que las semillas sombra-intolerantes de las invasoras no se establezcan, pero sí las especies endémicas (Arellano 2012).

$\mathrm{El}$ viento en la isla $\mathrm{RC}$ es frecuente con dirección predominante sur-este, siendo especialmente intensos desde fines del verano hasta otoño (Hajek \& Espinoza 1987), época que coincide con la fructificación de los arbustos invasores. En este escenario, la dispersión de frutos carnosos por el viento podría ser un fenómeno frecuente. Zúñiga et al. (2011') instalaron trampas de semillas en PEY para discernir el rol en la dispersión de semillas de estos dos agentes. Fue posible distinguir la dispersión por zorzal de la de viento, debido a que en el primer caso se dispersan semillas y en el segundo se dispersan frutos. Al conocer la especie de árbol sobre el cual está la trampa de semillas se descarta la posible dispersión por gravedad (barocoría). En claros de bosque, el ambiente donde germinan las plantas invasoras, no había árboles sobre las trampas de semillas, por lo cual es posible distinguir la dispersión por viento de la realizada por aves.

1 ZÚÑIGA J, D TABILO \& C SMITH-RAMÍREZ (2011) Effects of Turdus on seed dispersal of invasive and native species on Robinson Crusoe Island. II Biolief Congress. Mar del Plata, Argentina. 
Los resultados muestran que la dispersión anemócora de frutos de maqui y por ende de semillas, con $2.04 \pm 0.01$ semillas por fruto (media \pm DE) entre fines de enero a fines de febrero, fue de $17.9 \%$ (8.16 semillas trampa ${ }^{-1}$; Fig. 1A). La dispersión endozoócora fue de $82.1 \%$ (38.85 semillas trampa-1; Fig. 1A). Estos resultados se refieren a semillas caídas en claros y bordes de claros, ambiente donde pueden germinar. La dispersión de semillas de mora, durante marzo, fue nula en claros (solo se encontraron semillas dispersadas en matorral invasivo y bosque aledaño). La dispersión de semillas entre abril a principios de julio, en claros y borde de claros, fue realizada por zorzal (Fig. 2B), no se encontró dispersión de semillas por viento. En la Fig. 3C se muestra el aporte por viento en la dispersión de semillas de una leñosa nativa intolerante a la sombra, Juan Bueno, la cual fructifica en otoño-invierno. En claros de bosque el aporte de semillas dispersadas por viento (dos semillas por fruto) y el zorzal fue similar a lo encontrado para maqui.

Debe considerarse que es probable que las semillas dispersadas por viento (en realidad frutos) tengan poca o nula capacidad de germinación a menos que su pericarpo y endocarpo sea liberado por la acción del viento, agua, suelo, otros, una vez que el fruto llega al piso del bosque. La inhibición de la germinación por la pulpa de los frutos es un fenómeno frecuente (Cipollini \& Levey 1997).

En conclusión, la mayor parte de la lluvia de semillas de maqui y mora en claros de bosque sería aportada por zorzal.

\section{3. ¿LA GERMINACIÓN DE LAS SEMILLAS DE LAS PLANTAS INVASORAS ES AFECTADA POR EL PASO A TRAVÉS DEL TRACTO DIGESTIVO DEL AVE MUTUALISTA?}

En fecas de zorzal colectadas en PEY en el verano 2010 se encontraron en promedio \pm $\mathrm{DE}$ de $5 \pm 5.2$ semillas de maqui y $5 \pm 1.1$ de mora, con un máximo de 24 y 66 semillas por feca, respectivamente (Abarzúa \& SmithRamírez 2010). En ensayos en laboratorio con semillas provenientes de estas fecas, se encontró que en promedio las semillas de maqui defecadas por zorzal presentaron diferencias positivas y significativas en su porcentaje de germinación respecto a las
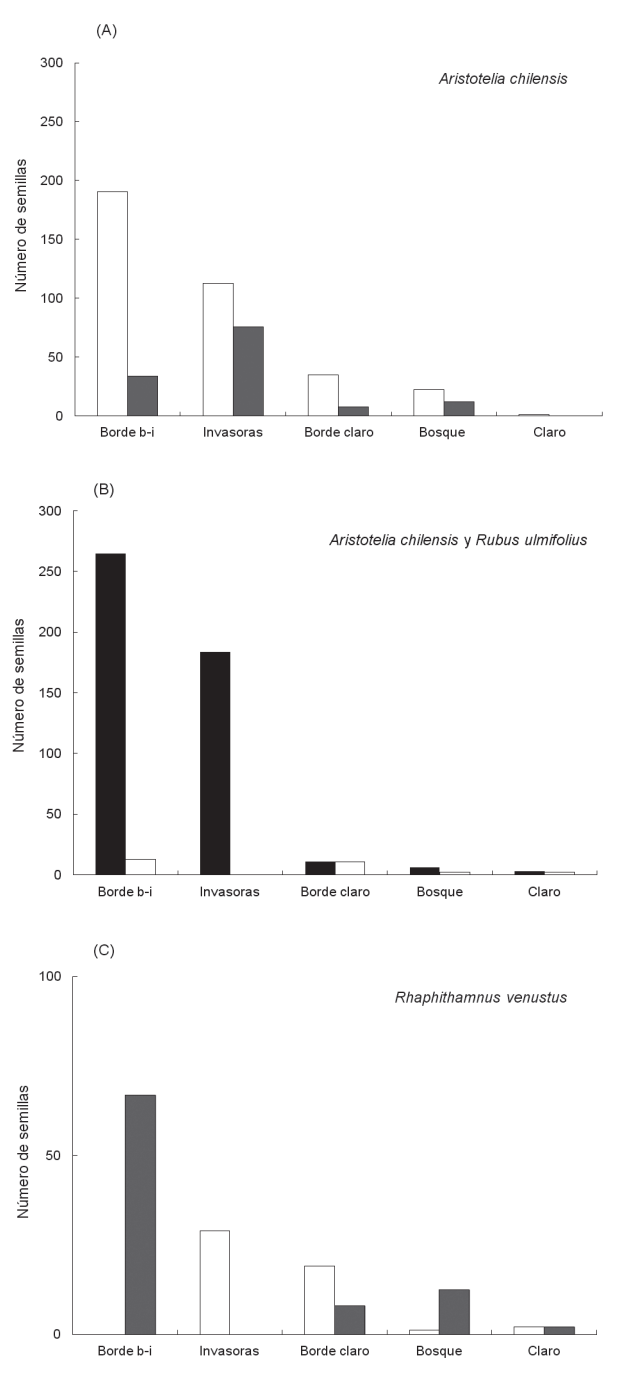

Figura 2: (A): Número total de semillas de Aristotelia chilensis caídas en trampas de semillas. (B): Número de semillas de Rubus ulmifolius (barra negra) y de Aristotelia chilensis (barra blanca) caídas en trampas de semillas. (C): Número total de semillas de Rhaphithamnus venustus caídas en trampas de semillas. El número de semillas fue estandarizado por número de trampas. Todas las trampas están ubicadas en el bosque de Plazoleta El Yunque. En blanco las semillas defecadas por Turdus falcklandii, en gris las semillas provenientes de frutos transportados por viento o gravedad. Borde $\mathrm{b}-\mathrm{i}=$ Borde del bosque con el matorral invasivo.

(A): Total seed numbers of Aristotelia chilensis collected in seed traps. (B): Total seed numbers of Rubus ulmifolius (black bar) and Aristotelia chilensis (white bar) collected in seed traps. (C): Seed number of Rhaphithamnus venustus collected in seed traps. Seed numbers were standardized by number traps. All the traps were in Plazoleta el Yunque forest. In white, seeds defecated by Turdus falcklandii, in grey, seeds dispersed by wind or gravity. Borde $\mathrm{b}-\mathrm{i}=$ Forest edge with invasive shrubland. 
extraídas manualmente de los frutos, promedio $\pm \mathrm{DE}$ de $22.5 \% \pm 7.2$ defecadas, versus $5 \% \pm 6$ extraídas manualmente. La germinación de las semillas de mora no fue facilitada al pasar por el tracto digestivo del zorzal, promedio $\pm \mathrm{DE}$ de $42.5 \% \pm 6$ defecadas y $50 \% \pm 9 \%$ extraídas manualmente. Por el contrario, las semillas de murta fueron desfavorecidas al pasar por el tracto digestivo de zorzal, promedio $\pm \mathrm{DE}$ de $84 \% \pm 6 \%$ defecadas y $96 \% \pm 1$ extraídas manualmente (Mora et al. 20112).

Análisis de 209 fecas colectadas en el verano 2010 muestran que el zorzal dispersa en un $92.6 \%$ semillas de maqui y mora, y solo $8.4 \%$ semillas de otras especies, probablemente nativas. Entre estas últimas se pudieron distinguir semillas de Azara serrata var. fernandenziana (Gay) Reiche y Berberis corymbosa Hook et Ar. En 102 fecas colectadas en otoño 2011, se encontró un $100 \%$ de semillas de especies invasoras (mora y murta), ninguna nativa. Cabe señalar que ambas colectas de fecas fueron realizadas en el ecotono de especies invasoras y bosque nativo, lo que pudo afectar la identidad y abundancia de las semillas encontradas. Hasta ahora no se conoce el efecto del zorzal sobre la germinación de semillas nativas de RC, pero es probable que en el caso de Drimys este efecto sea nulo, debido a que las semillas de este género responden principalmente a escarificación por frío y mecánica (Cabello \& Botti 1987). Además, el embrión de las semillas del género Drimys madura después que su pericarpo, necesitando 3-9 meses de escarificación en frío para germinar.

\section{4. ¿CAMBIARÁ A NIVEL COMUNITARIO Y DE}

PAISAJE LA ABUNDANCIA Y DISTRIBUCIÓN DE ESPECIES DE PLANTAS INVASORAS DISPERSADAS

POR EL AVE MUTUALISTA SI ESTE FUERA CONTROLADO O ERRADICADO?

Debe tenerse presente que al controlar la población del principal dispersor de semillas

2 MORA JP, C SMITH-RAMÍREZ \& RE COOPMAN (2011) Efecto del paso de semillas de la planta invasora, Ugni molinae, a través del tracto digestivo de un ave nativa, Turdus falcklandii, en la isla Robinson Crusoe. XVIII Reunión Anual de la Sociedad de Ecología de Chile, Noviembre 2011, Puerto Varas, Chile. introducidas, lo que se está haciendo es disminuir la lluvia y sombra de semillas, pero no a los individuos adultos de las plantas invasoras. A partir de datos de germinación en terreno de semillas, densidad de plántulas y juveniles de maqui y mora en 37 claros de bosque (Arellano 2012), y estimaciones de la densidad de individuos adultos, Castillo et al. $\left(2011^{3}\right)$ realizaron curvas demográficas de las especies invasoras (Fig. 3). Para maqui, la curva demográfica se asemeja al tipo II, que implica una tasa relativamente constante de mortalidad independiente de la fase o edad. Mientras que para mora se asemeja a la de tipo III, indicando una alta pérdida en las primeras fases o edades, seguida por un periodo de pérdidas menores (Castillo et al. 2011). De esta curva de supervivencia se desprende que de 250 semillas del banco de semillas transitorio de maqui y de 9000 semillas del banco de semillas de mora se producirá un individuo adulto. En ambas especies la mayor mortalidad en el ciclo de vida ocurre entre la fase semilla a plántula establecida (Fig. 3). Esta mortalidad ocurriría principalmente por dos razones: 1.- Baja tasa de germinación y pérdida de viabilidad de las semillas. Las semillas de maqui, así como de otras especies del género Aristotelia, son recalcitrantes (Rowarth et al. 1999), es decir, no forman banco de semillas permanente. Al parecer las semillas de Rubus ulmifolius tienen baja viabilidad inter-anual (Piudo \& Cavero 2007), no sobrepasando los dos años (Mora et al. datos no publicados). 2.- Depredación, realizada por invertebrados, al parecer babosas (Gastropoda: Pulmonata) e insectos no identificados (obs. pers. de los autores). Estos dos factores disminuyen considerablemente la cantidad de plántulas de invasoras que pasan a la etapa juvenil en claros del bosque de PEY. Probablemente la herbivoría por roedores y conejos también afectaría el paso de la fase plántula a juvenil.

La densidad de la lluvia de semillas de maqui, estimada a partir de los datos de

3 CASTILLO J, C SMITH-RAMÍREZ, G ARELLANO, J MORA \& H GUTIÉRREZ (2011) Demografía de dos especies invasoras: Aristotelia chilensis y Rubus ulmifolius en claros del bosque nativo en la isla Robinson Crusoe, Chile. XVIII Reunión Anual de la Sociedad de Ecología de Chile, noviembre 2011, Puerto Varas, Chile. 
TABLA 2

Especies de plantas de las islas Robinson Crusoe y Alejandro Selkirk que presentan frutos dispersados por aves, según Bernardello et al. (2006). En negrita se muestran las especies que han sido observadas de ser dispersadas por Turdus falcklandii, o cuyas semillas han sido encontradas en sus fecas. Se excluyen de esta lista las especies extintas endozoócoras, además de Greigia berteroi, la cual nunca ha sido vista en flor. Gunnera, Peperomia y Ochagavia tienen fruto carnoso, pero es improbable que sean dispersadas por Turdus, debido a lo reducido de su tamaño $(<1.5 \mathrm{~mm})$. E = endémico, $\mathrm{N}$ = nativo, Hierba $\mathrm{p}=$ hierba perenne.

Plant species of Robinson Crusoe and Alejandro Selkirk Islands with bird dispersed fruits, sensu Bernardello et al. (2006). Species in bold are those dispersed by Turdus, confirmed by direct observation or presence of seeds in feces. Species exclude from this list are extinct endozoochorus species and Greigia berteroi, which was never observed in flowering. Gunnera, Peperomia and Ochagavia have fleshy fruit, but are not likely dispersed by Turdus, due to small diameter $(<1.5 \mathrm{~mm})$. $\mathrm{E}=$ endemic, $\mathrm{N}$ = native, Hierba $\mathrm{p}=$ perennial herb.

\begin{tabular}{lccccc}
\hline Especie & Familia & Origen & Hábito & $\begin{array}{c}\text { Tipo de } \\
\text { fruto }\end{array}$ & $\begin{array}{c}\text { Síndrome } \\
\text { de dispersión }\end{array}$ \\
\hline Robinson Crusoe & & & & & \\
Berberis corymbosa & Berberidaceae & $\mathrm{E}$ & Arbusto & Baya & Endozoocoría \\
Pernettya rigida & Ericaceae & $\mathrm{E}$ & Arbusto & Baya & Endozoocoría \\
Azara serrata & Flacourtiaceae & $\mathrm{E}$ & Árbol & Baya & Endozoocoría \\
Myrcengenia fernandeziana & Myrtaceae & $\mathrm{E}$ & Árbol & Baya & Endozoocoría \\
Ugni selkirkii & Myrtaceae & $\mathrm{E}$ & Arbusto & Baya & Endozoocoría \\
Colletia spartioides & Rhamnaceae & $\mathrm{E}$ & Hierba & Drupa & Endozoocoría \\
Margyricarpus digynus & Rosaceae & $\mathrm{E}$ & Hierba & Baya & Endozoocoría \\
Solanum pentadlii & Solanaceae & $\mathrm{E}$ & Hierba & Baya & Endozoocoría \\
Solanum fernandezianum & Solanaceae & $\mathrm{E}$ & Hierba & Baya & Endozoocoría \\
Rhaphithamnus venustus & Verbenaceae & $\mathrm{E}$ & Árbol & Baya & Endozoocoría \\
Drimys confertifolia & Winteraceae & $\mathrm{E}$ & Árbol & Baya & Endozoocoría \\
Alejandro Selkirk & & & & & \\
Empetrum rubrum & Empetraceae & $\mathrm{N}$ & Arbusto & Baya & Endozoocoría \\
Myrceugenia schulzei & Myrtaceae & $\mathrm{E}$ & Árbol & Baya & Endozoocoría \\
Myrteola nummularia & Myrtaceae & $\mathrm{N}$ & Arbusto & Baya & Endozoocoría \\
Rubus geoides & Rosacaeae & $\mathrm{N}$ & Hierba p & Baya & Endozoocoría \\
Nertera granadensis & Rubiaceae & $\mathrm{N}$ & Hierba p & Baya & Endozoocoría \\
\hline
\end{tabular}

trampas de semillas (de $30 \mathrm{~cm}$ de diámetro) de Zúñiga et al. (2011) en PEY, fue: zorzal = 0.19 semillas $\mathrm{m}^{2}$ día ${ }^{-1}$; viento $=0.04$ semillas $\mathrm{m}^{2}$ día $^{-1}$. Es decir, estimamos que durante enero de cada año en promedio \pm DE de $23( \pm 11.2)$ semillas diarias de maqui son depositadas en un claro de $100 \mathrm{~m}^{2}$ de PEY, los cuales están a una distancia promedio de 30 metros del matorral invasor (Arellano 2012). Análisis a nivel de paisaje revelan que a medida que es mayor la distancia a la fuente de propágulos, menor es la densidad de propágulos de invasoras que se encuentran en los claros de PEY (Arellano
2012) y en general en toda la superficie boscosa de RC (Díaz 2012). Otros antecedentes como el tiempo de tránsito de las semillas por el tracto digestivo, la velocidad de desplazamiento del ave y la distancia a la fuente de propágulos permitirían determinar una función o "kernel" de dispersión en función del paisaje. Existen modelos de manejo referidos a la dispersión de semillas en paisajes simulados (With 2002, Gosper et al. 2005). Sin embargo, modelos de dispersión que incorporen todas estas variables, incluyendo probabilidades de transición demográfica de las plantas invasoras y de tasas 


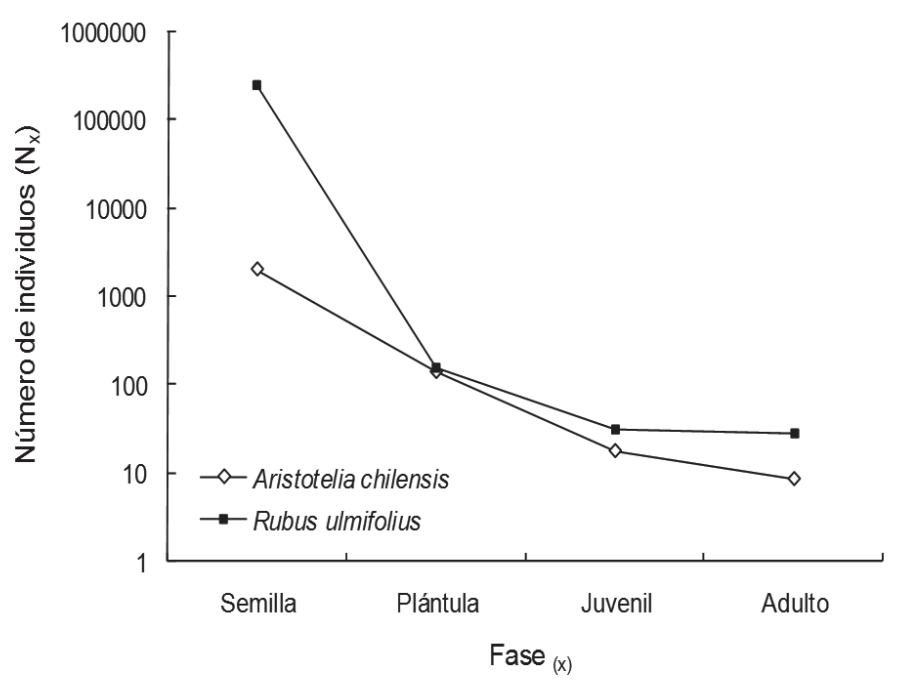

Figura 3. Curvas de supervivencia de Aristotelia chilensis y Rubus ulmifolius en claros del bosque de Plazoleta el Yunque de la isla Robinson Crusoe, Juan Fernández, Chile.

Survival curve of Aristotelia chilensis and Rubus ulmifolius in gap forests in Plazoleta el Yunque, Robinson Crusoe Island, Juan Fernández, Chile.

reproductivas de las aves dispersoras, resultan necesarios para determinar cuántos individuos de zorzal deberían ser controlados anualmente para disminuir la lluvia de propágulos de las especies invasoras, o bien si esta especie debe ser erradicada del sistema para producir cambios demográficos en las nuevas cohortes de invasoras.

\section{SI EL AVE ES CONTROLADA O ERRADICADA ¿PRODUCIRÁ CAMBIOS NEGATIVOS EN INTERACTUANTES QUE NO SEAN LAS PLANTAS INTRODUCIDAS?}

Como se muestra en la Fig. 2C, el zorzal dispersa semillas de especies nativas (en este caso Juan Bueno). Datos no publicados por los autores de este trabajo muestran que también dispersa en alta proporción las semillas de canelo y en baja abundancia las semillas de luma. La escasez de semillas nativas en fecas (como se señala en la pregunta 3), en trampas de semillas (a excepción de canelo y Juan Bueno) y la falta de sitios de establecimiento donde las nativas puedan sobrepasar la capacidad de crecimiento de las invasoras, nos permiten postular que el efecto de quitar al zorzal del sistema no traerá efectos negativos superiores a los que existen al estar presente.
Por otra parte, el zorzal ejerce un rol de predador de insectos introducidos en las cercanías del poblado San Juan Bautista, como son los Curculiónidos: Otiorhynchus rugosostriatus Goeze (13 \% de restos, $\mathrm{n}=$ 209 fecas de verano estudiadas), Naupactus leucoloma Boheman (5 \% de restos, $\mathrm{n}=209$ fecas examinadas) y el Hymenoptero: Vespula germanica Fabricius (2\% de restos, $\mathrm{n}=209$ fecas) y de otras especies introducidas con menos de $1 \%$ de restos (Muñoz et al. datos no publicados). Además, el zorzal también consume artrópodos nativos, como miriápodos, crustáceos y caracoles terrestres endémicos (Muñoz et al. datos no publicados, obs. pers. de los autores). En otoño estaría consumiendo preferentemente frutos de murta y no artrópodos (datos no publicados de los autores). El efecto de controlar o erradicar al zorzal sobre la completa trama de interacciones en que participa es complejo de conocer, y probablemente se escapa a cualquier estudio tradicional de manejo. Las medidas de manejo, sin embargo, no requieren un conocimiento completo del sistema, el cual muchas veces es imposible. Cuando se maneja se asumen riesgos, en que se sopesan los beneficios frente a los probables problemas que este manejo pueda suscitar. 


\section{DISCUSIÓN}

Las primeras cuatro preguntas planteadas sobre lo que se debe tener conocimiento para tomar decisiones de manejo en relación a mutualistas dispersores nativos y especies invasoras, incluyen todos los aspectos abordados en Buckley et al. (2006), pero además incorpora una quinta pregunta que trata sobre la complejidad trófica de las interacciones que establece un dispersor omnívoro, como es el caso del zorzal. Además, la cuarta y quinta pregunta se enfocan en plantear los efectos de las medidas de manejo del ave mutualista sobre el sistema de interés.

Una medida para controlar el avance de las plantas invasoras (ya antes puesta en práctica por CONAF y actualmente por la Municipalidad del AJF), ha sido disminuir la lluvia de semillas al interior del bosque al controlar la población del dispersor. Lamentablemente, cuando se realizó esta medida hace once años, no se cuantificó si ello produjo una disminución de la lluvia de semillas de las especies invasoras, o de la invasión en claros. Debe considerarse que si el zorzal fuera fuertemente controlado o erradicado de la isla RC, la expansión de maqui, mora y murta no se detendrá, aunque sí se desacelerará. La reproducción vegetativa de las plantas invasoras continuará ganando espacio $\mathrm{y}$ otros agentes de dispersión no dejarían de estar presentes como el viento y quizás otras especies introducidas como el coatí (Nasua nasua Linnaeus) y las ratas. Estas especies son responsables de dispersar semillas pequeñas en el neotrópico y en Hawaii, respectivamente (Alves-Costa \& Eterovick 2007, Medeiros 2004). Por otra parte, planes para erradicar estos mamíferos introducidos se encuentran en marcha en el AJF (Saunders et al. 2011).

Respecto a las preguntas planteadas inicialmente, podemos decir que la sentencia de Dirnbock et al. (2003) y Honeyman et al. (2005) parece cumplirse en los análisis a microescala realizados recientemente por Arellano (2012) y por los datos de tasa anual de generación de claros mostrada en este trabajo. Si bien se cuenta con solo un año de datos en un solo sector de la isla para poder establecer el área anual potencial a ser invadida, pensamos que sumado a las evidencias de otros autores, es más que preocupante el fenómeno invasivo de plantas en el AJF. A través de los antecedentes preliminares mostrados en la Fig. 2, podemos decir que el zorzal es el principal dispersor de semillas de maqui y mora. Los antecedentes respecto a murta provienen de la presencia de numerosas semillas en fecas de zorzal. Pensamos que el efecto de, un aumento o disminución, en la capacidad de germinación de las semillas de invasoras producto de la ingesta por zorzal, sería insignificante respecto a la producción de individuos adultos, debido a la gran cantidad de lluvia de semillas producidas por las especies invasoras.

La demografía de las plantas invasoras más los antecedentes de densidad de lluvia de semillas y la viabilidad del banco de semillas, arrojan valiosa información que permitiría modelar el efecto de controlar al zorzal sobre la densidad de lluvia de semillas y en fases posteriores del ciclo de vida. Así, de los datos de lluvia de semillas y a partir de información de tablas de vida de invasoras, se desprende que existe una sobre-dispersión de semillas y un banco de semillas excedido en relación al número de plántulas, juveniles y adultos de las especies invasoras.

Por otra parte, en relación a la pregunta cinco, es incierto el cambio que experimentará la abundancia y distribución de las plantas nativas dispersadas por el zorzal, si este fuera controlado o erradicado temporalmente. Sin embargo, el cambio de cobertura en la comunidad de especies nativas por la expansión de plantas invasoras es alto, resultando en pérdidas de biodiversidad. Las medidas de control o erradicación del ave dispersora de semillas deben ser ponderadas con respecto a otras medidas de control de las plantas invasoras. En este sentido, la experiencia de la comunidad fernandeziana, luego de siete años controlando mecánica y químicamente maqui y mora, muestra que esta actividad es eficaz, ya que permite reestablecer la regeneración arbórea, cobertura y riqueza de especies nativas en los sitios despejados. Esta actividad de despeje va acompañada de la extracción anual de los nuevas plántulas de maqui y mora que se generan producto de la dispersión de semillas o el banco de semillas de mora. El control de una hectárea de maqui y mora en $\mathrm{RC}$ tarda entre 3.6 a 8.7 meses realizado por un equipo de cinco personas (existen 805.9 ha de estas especies). El costo por controlar una hectárea de maqui y mora cerca del poblado 
son 21,6 millones de pesos considerando honorarios y material, en un tiempo mínimo de 3.6 meses (datos de los autores basados en el control realizado durante siete años por Oikonos). Aún no se sabe cómo controlar murta. Probablemente tomaría cincuenta años, al menos, controlar significativamente estas especies y una cantidad de dinero considerable. Los cálculos anteriores no consideran el aumento poblacional de las especies invasoras mientras se controlan. Además, dado que la mayor parte de la isla $\mathrm{RC}$ tiene más de $25^{\circ}$ de pendiente, probablemente no sea posible erradicarla, sino solo controlarla. Estas dificultades en controlar las especies invasoras han llevado a varios científicos a considerar que no es posible recuperar la biodiversidad de la isla. Por otro lado, no se sabe el costo ni eficacia de metodologías de control o erradicación del zorzal, dado que no hay antecedentes sobre erradicación de esta especie, ni de alternativas de metodologías adecuadas para ser utilizada en una campaña (Saunders et al. 2011) salvo la experiencia relativamente exitosa realizada por CONAF. Expertos consideran que un intento de erradicación en este momento tiene una alta probabilidad de fracaso y recomiendan estudiar la ecología del zorzal y diferentes técnicas de remoción antes de considerar un control o erradicación (Saunders et al. 2011). Creemos que si el zorzal fuese una especie endémica a Juan Fernández se podrían suscitar problemas éticos de controlarlo o erradicarlo, pero siendo al parecer una especie nativa, como lo demuestra cierta diferenciación genética encontrada en Selkirk, no debiera presentar problemas de este tipo. Si fuese una especie introducida no presentaría más problemas que el control que se hace de la cabra (Capra aegragus Erxleben), especie introducida que ha llegado a considerarse por sus características morfológicas como una subespecie de Juan Fernández.

El problema de la expansión de plantas invasoras en islas ocurre a nivel mundial, no habiendo una clara forma de detener este avance. Por ejemplo, en la isla Reunión, $R$. alceifolius Poir y en Galápagos $R$. niveus Thunb. son reconocidas como agresivas invasoras (Baret et al. 2008). En Galápagos, Rubus llegó hace 40 años, habiendo monopolizado 30000 ha de las islas Isabela, Floreana y Santiago, con un potencial de ocupar 90000 ha más. Anualmente la Dirección del Parque Nacional de Galápagos invierte USD 200000 para el control de 160 ha, menos del $1 \%$ del área total impactada, mientras $R$. niveus continúa expandiéndose a ritmo acelerado. Debido a los altos costos de controlar esta especie, se busca en Galápagos un control biológico, este control suele ser un hongo o insecto que habita en el rango natural de la especie. El encontrar y probar un control biológico puede costar de USD 1 a 10 millones de dólares y hasta 10 años para obtener el método apropiado (Atkinson et al. 2010). En la isla Reunión se encontró un controlador biológico para $R$. alceifolius, en Hawaii cinco controladores biológicos (insectos defoliadores) fueron liberados entre 1969-1986. Estos desfoliadores atacaron tanto plantas invasoras como especies nativas, por lo cual, el plan de control biológico se detuvo (Atkinson et al. 2010). En el AJF como en otros casos, nada asegura que un control biológico de $R$. ulmifolius dé resultado. Al parecer el candidato más idóneo sería el hongo Phragmidium violaceum (Schulz) G. Winter, el cual está siendo estudiado para controlar a $R$. niveus en Galápagos (Atkinson et al. 2010). Debido a la alta tasa de colonización de maqui, mora y murta, la búsqueda de un control biológico debe ir en paralelo a la lenta pero efectiva remoción mecánica de estas plantas, especialmente en los sitios donde existen poblaciones de alguna de las 25 especies de plantas en estado crítico de extinción (CONAF 2009).

La decisión de controlar o erradicar al principal agente dispersor de semillas, es una decisión de tipo político y ético, basado en la opinión pública y en los antecedentes científicos. Al respecto el Sr. Hugo Arnal de Island Conservation señala: "El control del nivel poblacional de una especie nativa o inclusive su eventual erradicación de un área de conservación, particularmente si es una isla, con el objeto de asegurar la conservación de otras especies y ecosistemas de alta prioridad de conservación, es una decisión que debe enmarcarse dentro de las políticas ambientales del país y debe estar sustentado por la mejor información científica existente y por los especialistas más reconocidos de los taxa y ecosistemas involucrados. Obviamente, si se trata de políticas ambientales con objetivos claramente definidos, que tocan un tema tan sensible y biológicamente complejo, los 
organismos de gobiernos responsables de tomar esta decisión deben prestarle atención a los intereses del público general. Además, lor organismos de gobierno deben desarrollar los mecanismos de información, consulta y participación que sean necesarios para asegurar que la decisión final no solo es científicamente correcta sino que es socialmente aceptable". Por otra parte, la opinión de la mayoría de los ciudadanos de RC y algunos ornitólogos nacionales, es que gran parte de la solución al problema de expansión de las plantas invasoras en el AJF se solucionaría si el Gobierno, específicamente el SAG, declarara "especie dañina” al zorzal y se procediera a su erradicación.

Finalmente, concluimos que los antecedentes aportados muestran que el zorzal es el principal agente dispersor de plantas invasoras en claros de bosque de RC. Por otra parte, consideramos que la modelación del efecto de controlar el zorzal sobre la producción de adultos de invasoras podría aportar valiosos antecedentes que permitan decidir si es necesario controlar o erradicar esta especie de RC. Cualquiera de estas dos medidas debiera ser tomada a corto plazo para evitar la colonización de invasoras a las áreas aún no invadidas. Además, en paralelo a cualquiera de estas decisiones se debieran realizar estrategias de control de plantas invasoras en todas las etapas de su ciclo de vida como una forma de detener su avance.

AGRADECIMIENTOS: Agradecemos las conversaciones con Iván Leiva, Hernán González, Paola González de Rodt, Philippe Danton y los guardaparques en general, cuyos valiosos comentarios dieron origen a gran parte de este documento. Agradecemos la elaboración de parte de la Tabla 2 a Dafne Gho y las numerosas revisiones aportadas por Juan Pablo Mora. Agradecemos especialmente la valiosa colaboración de dos revisores anónimos y al Editor Asociado de esta revista. Además agradecemos las palabras aportadas a este artículo por Hugo Arnal de Island Conservation. La investigación y redacción de este documento fue financiada por UNESCO-MAB 2009, CONAF, por el Centro de Estudios Avanzados en Ecología y Biodiversidad (CASEB) de la Universidad Católica, Rufford Small Grant y por el Instituto de Ecología y Biodiversidad (IEB). A la fecha de publicación de este documento, el zorzal del AJF ya fue clasificado por el Servicio Agrícola y Ganadero, como especie dañina para su biodiversidad.

\section{LITERATURA CITADA}

ABARZÚA A \& C SMITH-RAMÍREZ (2010) Informe Unesco-MAB program, proyecto: Establishing the bases for a long term study about endemic biodiversity in Juan Fernandez Archipelago, Chile, Valdivia, Chile.

ALVES-COSTA C \& P ETEROVICK (2007) Seed dispersal services by coatis (Nasua nasua, Procyonidae) and their redundancy with other frugivores in southeastern Brazil. Acta Oecologica 32: 77-92.

ARELLANO GS (2012) Evaluación de la dinámica de invasión de Aristotelia chilensis (Elaeocarpaceae) y Rubus ulmifolius (Rosaceae) en claros de dosel en un bosque de la isla Robinson Crusoe, Archipiélago de Juan Fernández, Chile. Tesis de Magíster, Áreas Silvestres y Conservación de la Naturaleza, Facultad de Ciencias Forestales y de la Conservación de la Naturaleza, Universidad de Chile, Santiago, Chile.

ARMESTO JJ, C SMITH-RAMIREZ \& C SABAG (1996) The importance of plant-bird mutualisms in the Temperate Rainforest of Southern South America. En: Lawford RG, PB Alaback, E Fuentes (eds) High latitude Rain Forest and associated ecosystem of the west coast of the American: 248265. Springer-Verlag, New York, USA.

ARROYO MK (1999) The Archipelago of Juan Fernandez. En: Mittermeier R, N Myers, P Robles Gil \& CG Mittermeier (eds) Hotspots: 173. Ediciones de Cemex, Ciudad de México, México.

ATKINSON R, A GUEZOU, JC GUZMAN, A LLERENA, M GARDENER \& JL RENTERIA (2010) Diagnóstico y planificación para el desarrollo de un agente de control biológico para Rubus niveus en las islas Galápagos. Informe Parque Nacional Galápagos y Fundación Charles Darwin. Galápagos, Ecuador.

BARET S, L COURNAC, C THEBAUD, P EDWARDS \& D RASBERG (2008) Effects of canopy gap size on recruitment and invasion on the non-indigenous Rubus alcelifolius in lowland tropical rain forest on Réunion. Journal of Tropical Ecology 24: 337-345.

BERGSTROM DM \& SL CHOWN (1999) Life at the front: history, ecology and change on southern ocean islands. Trends in Ecology and Evolution 14: 472-477.

BERNARDELLO G, GJ ANDERSON, TF STUESSY \& DJ CRAWFORD (2006) The angiosperm flora of the Archipelago Juan Fernandez (Chile): Origin and dispersal. Canadian Journal of Botany 84: 12661281.

BROKAW N \& RT BUSING (2000) Niche versus chance and tree diversity in forest gaps. Trends in Ecology and Evolution 15: 183-188.

BUCKLEY Y, S ANDERSON, CP CATTERALL, RT CORLETT, T ENGEL et al. (2006) Management of plant invasions mediated by frugivore interactions. Journal of Applied Ecology 43: 848857.

CABELLO A \& J BOTTI (1987) Estudio de germinación en Drimys winterii Forst. (Canelo). Revista de Ciencias Forestales 5: 21-30.

CASTRO SA, M MUÑOZ \& FM JAKSIC (2007) Transit towards floristic homogenization on oceanic islands in the south-eastern Pacific: Comparing pre-European and current floras. Journal of Biogeography 34: 213-222. 
CASTRO SA \& FM JAKSIC (2008) Role of nonestablished plants in determining biotic homogenization patterns in Pacific Oceanic Islands. Biological Invasions 10: 1299-1309.

CIPOLLINI ML \& DJ LEVEY (1997) Secondary metabolites of fleshy vertebrate-dispersed fruits: Adaptive hypotheses and implications for seed dispersal. American Naturalist 150: 346-372.

CONAF (1976) Plan de manejo Parque Nacional Juan Fernández. Documento técnico de trabajo $\mathrm{N}^{\circ} 22$. Corporación Nacional Forestal. Santiago, Chile.

CONAF (2009) Plan de manejo Parque Nacional Archipiélago de Juan Fernández. Corporación Nacional Forestal, Santiago, Chile.

CUEVAS JG \& G VAN LEERSUM (2001) Project "conservation, restoration and development of the Juan Fernández Islands, Chile”. Revista Chilena de Historia Natural 74: 899-910.

CUEVAS JG, A MARTICORENA \& LA CAVIERES (2004) New additions to the introduced flora of the Juan Fernandez Islands: Origin, distribution, life history traits and potential of invasion. Revista Chilena de Historia Natural 77: 523-538.

CUEVAS J \& R VARGAS (2006) Drimys confertifolia Phil. Isla Robinson Crusoe. En: Donoso C (ed) Las especies arbóreas de los bosques templados de Chile y Argentina: 593-598. Ediciones Marisa Cuneo, Valdivia. Chile. Trama Impresiones S.A. Concepción. Chile.

DAEHLER CC, JS DENSLOW, S ANSARI \& HC KUO (2004) A risk-assessment system for screening out invasive pest plants from Hawaii and other Pacific islands. Conservation Biology 18: 360-368.

DALLING JW \& SP HUBBELL (2002) Seed size, growth rate and gap microsite conditions as determinants of recruitment success for pioneer species. Journal of Ecology 90: 557-568.

DANTON PH (2004) Plantas silvestres de la isla Robinson Crusoe. Guía de reconocimiento. Corporación Nacional Forestal, Valparaíso, Chile.

DANTON PH \& CH PERRIER (2006) Nouveau catalogue de la flore vasculaire de l'archipel Juan Fernández (Chili). Acta Botanica Gallica 153: 399-587.

DAVIS MA (2003) Biotic globalization: Does competition from introduced species threaten biodiversity? BioScience 53: 481-489.

DEBUSSCHE M \& M ISENMANN (1994) Birddispersed seed rain and seedling establishment in patchy Mediterranean vegetation. Oikos 69: 414-426.

DENSLOW JS (2003) Weeds in paradise: Thoughts on the invasibility of tropical islands. Annals of the Missouri Botanical Garden 90: 119-127.

DÍAZ I (2013) Análisis y modelación de la evolución espacio-temporal de la invasión de Rubus ulmifolius, Aristotelia chilensis y Ugni molinae, en la Isla Robinson Crusoe. Tesis, Ingeniería en Recursos Naturales, Facultad de Ciencias Agronómicas, Universidad de Chile, Santiago, Chile.

DIRNBÖCK T, J GREIMLER, P LOPEZ \& TF STUESSY (2003) Predicting future threats to the native vegetation of Robinson Crusoe Island, Juan Fernandez Archipelago, Chile. Conservation Biology 17: 1650-1659.

ELIAS RB \& E DIAS (2009) Gap dynamics and regeneration strategies in Juniperus-Laurus forests of the Azores Islands. Plant Ecology 200: 179-189.
FRITTS TH \& GH RODDA (1998) The role of introduced species in the degradation of Islands ecosystems: A case history of Guam. Annual Review of Ecology and Systematics 29: 113-140.

GASTON KJ, AG JONES, C HÄNEL \& SL SHOWN (2003) Rates of species introduction to a remote oceanic island. Proceedings of the Royal Society 270: 1091-1098.

GOSPER CR, CD STANSBURY \& G VIVIAN-SMITH (2005) Seed dispersal of fleshy- fruited invasive plants by birds: Contributing factors and management options. Diversity and Distributions 11: 549-558.

GREIMLER J, TF STUESSY, U SWENSON, CM BAEZA \& O MATTHEI (2002) Plant invasions on an oceanic archipelago. Biological Invasions 4: 73-85.

GRAVES WG, BB PECKHAM \& J PASTOR (2006) A 2D differential equations model for mutualism. Department of Mathematics and Statistics Technical Report. EditionsUniversity of Minnesota, Duluth.

HAHN I (2006) First reproductive records and nest sites of the endemic Juan Fernández Tit-tyrant Anairetes fernandezianus (Philippi, 1857) (Aves: Passeriformes: Tyrannidae) from Robinson Crusoe Island, Chile. Zoologische Abhandlungen (Dresden) 55: 177-190.

HAHN I, PM VERGARA \& U ROMER (2011) Habitat selection and population trends in terrestrial bird species of Robinson Crusoe Island: Habitat generalists versus forest specialists. Biodiversity and Conservation 20: 2797-2813.

HAJEK E \& GA ESPINOZA (1987) Meteorología, climatología y bioclimatología de las islas oceánicas chilenas. En: Castilla JC (ed) Islas oceánicas chilenas: Conocimiento científico y necesidades de investigación: 55-83. Ediciones Universidad Católica de Chile, Santiago, Chile.

HARTSHORN G (1990) Gap-phase dynamics and tropical tree species richness. En: Holm-Nielsen LB, IC Nielsen \& H Balslev (eds) Tropical forests - Botanical dynamics, speciation and diversity: 6573. Academic Press, London.

HONEYMAN P, P CRUZ \& C SCHULZE (2005) Estudio de los factores bióticos asociados a la regeneración del bosque de Juan Fernández y propuesta de restauración. Informe Técnico, Universidad Mayor y Corporación Nacional Forestal, Valparaíso, Chile.

HUBBELL S \& RB FOSTER (1986) Canopy gaps and the dynamics of a Neotropical forest. En: Crawley MJ (ed) Plant ecology. Blackwell Scientific Publications, Oxford.

JORDANO P \& EW SCHUPP (2000) Determinants of seed dispersal effectiveness: The quantity component in the Prunus mahaleb-frugivorous bird interaction. Ecological Monographs 70: 591615.

KUEFFER C, L KRONAUER \& PJ EDWARDS (2009) Wider spectrum of fruit traits in invasive than native floras may increase the vulnerability of oceanic islands to plant invasions. Oikos 118: 1327-1334.

KUEFFER C, CC DAEHLER, CW TORRES-SANTANA, C LAVERGNE, JY MEYER, R OTTO \& L SILVA (2010) A global comparison of plant invasions on oceanic islands. Perspectives in Plant Ecology, Evolution and Systematics 12: 145-161. 
LOOSER G (1927) La zarzamora (Rubus ulmifolius, Schoot) en Juan Fernández. Revista Chilena de Historia Natural 31: 84-85.

MANDON-DALGER I, P CLERGEAU, JN TASSIN, J RIVIERE \& S GATTI (2004) Relationships between alien plants and an alien bird species on Reunion Island. Journal of Tropical Ecology 20: 635-642.

MEDEIROS AC (2004) Phenology, reproductive potential, seed dispersal and predation, and seedling establishment of three invasive plant species in a Hawaiian rain forest. Ph.D. Dissertation, Department of Zoology, University of Hawaii at Manoa. Honolulu, USA.

MOONEY HA \& RJ HOBBS (2000) Invasive species in a changing world. Island Press, Washington DC, USA.

PIUDO MJ \& RY CAVERO (2007) Banco de semillas del suelo y composición florística de un carrascal de Navarra (España). Bulletin Societe Historie Naturalle (Toulouse) 141: 215-220.

RICHARDSON DM, N ALLSOPP, CM D'ANTONIO, SJ MILTON \& M REJMÁNEK (2000) Plant invasions - The role of mutualisms. Biological Reviews 75: 65-93.

ROWARTH JS, JG HAMPTON \& MJ HILL (1999) New Zealand native seed germination requirements: A Review. New Zealand Journal of Botany 45: 485-50.

TOMASEVIC J, MV LÓPEZ-CALLEJAS \& C ESTADES (2006) Estimación poblacional picaflor de Juan Fernández, temporada 2006. Informe interno Oikonos, California, USA.

SALA OE, F STUART CHAPIN III, JJ ARMESTO, E BERLOW, J BLOOMFIELD et al. (2000) Global biodiversity scenarios for the year 2100 . Science 287: 1770-1774.

SAX DF, SD GAINES \& JH BROWN (2002) Species invasions exceed extinctions on islands worldwide: A comparative study of plants and birds. The American Naturalist 160: 766-783.

SAUNDERS A, A GLEN, K CAMPBELL, R ATKINSON, J SAWYER, E HAGEN \& H TORRES (2010) Estudio sobre la factibilidad del manejo de especies invasoras en el archipiélago de Juan Fernández, Chile. Informe Invasive Species International, Island Conservation, Santiago, Chile

SIMBERLOFF D \& B VON HOLLE (1999) Positive interactions of nonindigenous species: Invasional meltdown? Biological Invasions 1: 21-32.

SKOTTSBERG C (1928) Pollinationsbiologie und samenverbreitung auf den Juan Fernandez-Inseln. En: Skottsberg C (ed) The natural history of the Juan Fernandez and Easter Islands: 2. 503-547.

SMITH-RAMIREZ C \& G ARELLLANO (2013) Necesidad de que las Fuerzas Armadas de Chile realicen control de plantas invasoras en el archipiélago de Juan Fernández, Chile. Bosque (en prensa).

STUESSY TF (1992) Diversidad de plantas en las islas Robinson Crusoe. En: Grau J \& G Ziska (eds) Flora silvestre de Chile: 54-66. Palmengarten Sonderheft 19, Frankfurt am Main, Alemania.

SWENSON U, TE STUESSY, M BAEZA \& DJ CRAWFORD (1997) New and historical plant introductions and potential pets in the Juan Fernandez Islands, Chile. Pacific Science 51: 233253.

THERY M (1989) Consommation des fruits et dissémination des graines par le Merlo noir (Turdus merula) en zone-ériurbaine sous climat tempéré. Acta Oecologia Applicata 10: 271-285.

VAN DER MEER PJ \& F BONGERS (1996) Patterns of tree-fall and branch-fall in a tropical rain forest in French Guiana. Journal of Ecology 84: 19-29.

VARGAS R, JG CUEVAS, C LE QUESNE, A REIF \& J BANNISTER (2010) Spatial distribution and regeneration strategies of the main forest species on Robinson Crusoe Island. Revista Chilena de Historia Natural 83: 349-363.

WILLIAMS P (2006) The role of blackbirds (Turdus merula) in weed invasion in New Zealand. New Zealand Journal of Ecology 30: 285-291.

WITH K A (2002) The landscape ecology of invasive species. Conservation Biology 16: 1192-1203.

YAMAMOTO SI (1992) The gap theory in forest dynamics. The Botanical Magazine, Tokyo 105: $375-383$. 